\title{
On Thermalisation of Energetic Charged Particles in Fusion Plasma with Quantum Electrodynamic Considerations
}

\author{
P.S. Ray and H. Hora \\ Department of Theoretical Physics \\ The University of New South Wales, Kensington, \\ New Sout Wales, Australia 2033 \\ (Z. Naturforsch. 32 a, 538-543 [1977] ; received March 21, 1977)
}

\begin{abstract}
The process of energy loss of charged heavy particles in plasma has been discussed taking into consideration photon emissions by electrons of plasma during scattering as is necessitated by quantum electrodynamics. Although the range in general increases with temperature, it is found that the numerical formula of former treatment giving a range as $T^{3 / 2}$ is not in general, obeyed. The range is susceptible to the initial energy as well as mass of the heavy particle as can be seen from the cases of alphas and reaction protons for DT, $\mathrm{DD}\left(\rightarrow \mathrm{p}+\mathrm{He}^{3}\right)$ and $\mathrm{D} \mathrm{He}^{3}$ plasmas.
\end{abstract}

\section{Introduction}

The penetration depth of alphas, protons or other $\mathrm{MeV}$-particles from nuclear reactions within thermonuclear plasmas is of importance for the reheat and total energy gain of these plasmas. In the case of tokamaks, the theory for deriving a more precise expression of the penetration depth has been elaborated by Düchs and Pfirsch ${ }^{1}$, where a remarkable improvement for the reactor conditions of tokamaks can be expected. The penetration depth plays an essential role for all concepts of fast-particle driven heating and (eventually compression) of plasmas of solid state density or higher. The decrease of the penetration depth by a factor 14 or more for very dense $\mathrm{MeV}$-electrons in $\mathrm{CO}_{2}$ plasma of solid state density compared to solid $\mathrm{CO}_{2}$ material, was a first indication of the strong differences ${ }^{2}$ to the usually known behaviour of solids. The easy explanation of these facts ${ }^{3}$ by using Bagge's generalization ${ }^{4}$ of the Bethe-Bloch-formula, encouraged us to evaluate the more general cases of alphas and other heavy charged particles in high density high temperature plasmas. An application to plasmas of the tokamak type will need more studies of additional forces, as e. g. incorporation of Lorentz-forces, etc.

Usually, the reheat in laser produced plasmas is calculated on the basis of a formula for the penetration depth $R$ derived by Winterberg ${ }^{5}$

$$
R \sim T^{3 / 2} .
$$

Reprint requests to Mrs. J. Stanic, Dept. of Theoretical Physics, University of New South Wales, Kensington, New South Wales, Australien 2033.
Winterberg's formula was used for the calculation of the nuclear fusion yields of inertial confinement of laser produced plasmas ${ }^{6}$ and in the general codes for laser compression by Brueckner and Jorna ${ }^{7}$. Though Brueckner himself was aware of the much more general properties of the penetration depth ${ }^{8}$.

As has been emphasized by Feynman ${ }^{9}$ it is impossible to scatter an electron with the emission of no photons. The real photon emissions in scattering brings with it the problem of infrared divergences of quantum electrodynamics and necessitates a cutoff factor and for this one has a natural estimate in plasma physics. One therefore has to reconstruct the Fokker-Planck formalism with this aspect. This is done in this work and also the explicit formula for the range has been derived. We recall that the energetic particles, e. g. alphas in DT-plasma have initial energies of some $\mathrm{MeV}$, whereas this plasma temperature is of the order of $\mathrm{keV}$. The motion of the heavy particle is therefore rectilinear until they lose energy by collision mainly with the electrons. The range can then be defined as the distance travelled until the energy is reduced to the thermal values.

It may be noted that in the work of Düchs and Pfirsch the Fokker-Planck formulation employed uses energy as a variable of the distribution function. A similar kinetic equation has been used by Tsui et al. ${ }^{10}$ in their work on the time development of the energy distribution function of the alphas. In contrast with these treatments, we are not evaluating the values of the distribution function, but we use more general cross sections and calculate the slowing down length $R$ of the alphas from the FokkerPlanck coefficients explicitly for numerical evaluation. 


\section{The Fokker-Plandk Formalism}

Consider a distribution of different species of particles denoted by indices $\mathrm{s}, \mathrm{t}, \ldots$ If $f_{\mathrm{s}}(r, v, t)$ is the distribution function for species s, its evolution is governed by the Fokker-Planck equation which we write in the form of Kramers

$$
\frac{\partial f_{\mathrm{s}}}{\partial t}+v_{i} \frac{\partial f_{\mathrm{s}}}{\partial x_{i}}+\frac{F_{i}}{m_{\mathrm{s}}} \frac{\partial f_{\mathrm{s}}}{\partial v_{i}}=\sum_{t} \sum_{n=1}^{\infty} \frac{(-1)^{n}}{n !} D^{(n)}\left(\alpha_{(n)}^{\mathrm{ts}} f_{\mathrm{s}}\right) \text {. }
$$

Here the sum extends over all species, $F_{i}$ is the $i$ th component of external force $(i=1,2,3)$ and we use the notation

$D^{(n)}=\frac{\partial^{n}}{\partial v_{i_{1}} \partial v_{i_{2}} \ldots \partial v_{i_{n}}}$; each $i_{k}$ takes values $1,2,3$. $a_{(n)}^{\mathrm{ts}}=\alpha^{\mathrm{ts}}\left(v_{i_{1}}, v_{i_{2}}, \ldots, v_{i_{n}}\right)$.

The $\alpha$ 's are the Fokker-Planck coefficients. One should note that thermodynamic equilibrium is not assumed for the validity of (2). We take for simplicity only two species of particles are present e. g. in a fusion plasma the electrons (subscript e) and the alphas (subscript a). We shall neglect the interaction among the alphas themselves. We further neglect the interaction $\alpha$-ions. To obtain the rate of energy loss of the alphas one has to go over to the hydrodynamic equations. Let $\psi(\boldsymbol{v})$ be a function of velocity $\boldsymbol{v}$ only. Its average $\bar{\psi}$ with respect to the distribution $f$ is defined as

$$
\bar{\psi}=\int \psi f \mathrm{~d} \boldsymbol{v} / \int f \mathrm{~d} \boldsymbol{v}=(\mathrm{l} / n) \int \psi f \mathrm{~d} \boldsymbol{v}
$$

where $d \mathbf{v} \equiv \mathrm{d} v_{1} \mathrm{~d} v_{2} \mathrm{~d} v_{3}$ and $n=n(\boldsymbol{r}, t)=\int f \mathrm{~d} \boldsymbol{v}$ the local density. Multiplying (2) by $\psi(\boldsymbol{v})$ and integrating with respect to $\mathrm{d} \boldsymbol{v}$ one obtains

$$
\begin{aligned}
\frac{\partial}{\partial t}\left(n_{\mathrm{a}} \bar{\psi}^{(\mathrm{a})}\right)+\frac{\partial}{\partial x_{i}} & \left(n_{\mathrm{a}}{\overline{\psi v_{i}}}^{(\mathrm{a})}\right)=\frac{1}{m_{\mathrm{a}}} n_{\mathrm{a}}{\frac{\overline{\partial\left(\psi F_{i}\right)}}{\partial v_{i}}}^{(\mathrm{a})} \\
& +\sum_{n=1}^{\infty} \frac{(-1)^{n}}{n !} \int \psi \boldsymbol{D}^{(n)}\left(\alpha_{(n)}^{\mathrm{ae}} f_{a}\right) \mathrm{d} \boldsymbol{v}
\end{aligned}
$$

where averaging is taken with respect to the alpha particle.

Now by partial integration one has

$$
\begin{aligned}
\int \psi D^{(n)}\left(\alpha_{(n)}^{\mathrm{ae}} f_{a}\right) \mathrm{d} \boldsymbol{v} & =(-1)^{n} \int \frac{\alpha_{(n)}^{\mathrm{ae}} D^{(n)} \psi f_{\mathrm{a}} \mathrm{d} \boldsymbol{v}}{} \\
& =(-1)^{n} n_{\mathrm{a}} \frac{\alpha_{(n)}^{\mathrm{ae}} D^{(n)} \psi}{(\mathrm{a})} .
\end{aligned}
$$

In the absence of external forces one can therefore write

$\frac{\partial}{\partial t}\left(n_{\mathrm{a}} \bar{\psi}^{(\mathrm{a})}\right)+\frac{\partial}{\partial x_{i}}\left(n_{\mathrm{a}} \overline{\psi v}_{i}^{(\mathrm{a})}\right)=\sum_{n=1}^{\infty} \frac{1}{n !} \overline{\alpha_{(n)}^{\mathrm{ae}} D^{(n)} \psi^{(\mathrm{a})}}$ where

$$
\alpha_{(n)}^{\mathrm{ae}} D^{(n)} \psi=\alpha^{\mathrm{ae}}\left(v_{i_{1}} v_{i_{2}} \ldots v_{i_{n}}\right) \frac{\partial^{n} \psi}{\partial v_{i_{1}} \partial v_{i_{2}} \ldots \partial v_{i_{n}}} \text {. }
$$

If one chooses $\psi(\boldsymbol{v})=\frac{1}{2} v^{2}=\frac{1}{2} \sum_{i=1}^{3} v_{i}^{2}$ so that all the derivitives of $\psi$ higher than second order vanish, one obtains:

$$
\begin{aligned}
\frac{1}{2} \frac{\partial}{\partial t}\left(n_{\mathrm{a}}{\overline{v^{2}}}^{(\mathrm{a})}\right)+ & \frac{1}{2} \frac{\partial}{\partial x_{i}}\left(n_{\mathrm{a}}{\overline{v^{2} v_{i}}}^{(\mathrm{a})}\right) \\
& =n_{\mathrm{a}}\left({\overline{a_{i} v_{i}}}^{(\mathrm{a})}+\frac{1}{2}{\overline{b_{i j} \delta_{i j}}}^{(\mathrm{a})}\right)
\end{aligned}
$$

where $a_{i}=\alpha\left(v_{i}\right)$ and $b_{i j}=\alpha\left(v_{i}, v_{j}\right)$ are the first two Fokker-Planck coefficients. To pick a particular alpha with coordinate and velocity $\left(\boldsymbol{r}_{\mathrm{a}}, \boldsymbol{v}_{\mathrm{a}}\right)$ we set

$$
f_{a}=\delta\left[\boldsymbol{r}-\boldsymbol{r}_{\mathrm{a}}(t)\right] \delta\left[\boldsymbol{v}-\boldsymbol{v}_{\mathrm{a}}(t)\right]
$$

giving $n_{\mathrm{a}}=\delta\left(\boldsymbol{r}-\boldsymbol{r}_{\mathrm{a}}\right)$ and $\overline{\psi(\boldsymbol{v})^{(\mathrm{a})}}=\psi\left(\boldsymbol{v}_{\mathrm{a}}\right)$. One will then obtain for the energy $E_{\mathrm{a}}=\frac{1}{2} m_{\mathrm{a}} v_{\mathrm{a}}{ }^{2}$ after integration with respect to $\boldsymbol{r}$ and $\boldsymbol{v}$ :

$$
\mathrm{d} E_{\mathrm{a}} / \mathrm{d} t=m_{\mathrm{a}}\left(\sum_{i} a_{i} v_{\mathrm{a}, i}+\frac{1}{2} \sum_{i} b_{i i}\right)
$$

for the rate of energy loss.

To evaluate further one has to substitute the values of $a_{i}$ and $b_{i i}$ as functions of $v_{i}$. Rosenbluth et al. ${ }^{11}$ have calculated these on the basis of Coulomb scattering and their result has been mostly utilised in plasma physics. Their calculation gives:

$$
\begin{aligned}
a_{i} & =\Gamma \frac{\partial}{\partial v_{i}} h(\boldsymbol{v}), \\
b_{i j} & =\Gamma \frac{\partial^{2}}{\partial v_{i} \partial v_{j}} g(\boldsymbol{v})
\end{aligned}
$$

where

$$
\begin{aligned}
& h(\boldsymbol{v})=\left(1+\frac{m_{\mathrm{a}}}{m_{\mathrm{e}}}\right) \int \mathrm{d} \boldsymbol{v}^{\prime} f_{\mathrm{e}}\left(\boldsymbol{v}^{\prime}\right) \frac{1}{\left|\boldsymbol{v}-\boldsymbol{v}^{\prime}\right|}, \\
& g(\boldsymbol{v})=\int \mathrm{d} \boldsymbol{v}^{\prime} f_{\mathrm{e}}\left(\boldsymbol{v}^{\prime}\right)\left|\boldsymbol{v}-\boldsymbol{v}^{\prime}\right|,
\end{aligned}
$$

and for $\Gamma$ ( $z=2$ for alpha)

$$
\Gamma=\left(4 \pi e^{4} z^{2} / m_{\mathrm{a}}{ }^{2}\right) \ln A
$$

with $A$ representing the Coulomb logarithm:

$$
\Lambda=\frac{3}{2} k T \frac{\lambda_{\mathrm{D}}}{e^{2}}=\frac{3}{4} \sqrt{\left(\frac{k T}{e^{2}}\right)^{3} \frac{1}{\pi N_{\mathrm{e}}}} .
$$

For the electrons we shall use the Maxwell-Boltzmann distribution

$$
f_{\mathrm{e}}(\boldsymbol{v})=N_{\mathrm{e}}\left(\frac{\gamma}{\pi}\right)^{3 / 2} e^{-\gamma v^{2}}
$$


where $N_{\mathrm{e}}$ is the electron density, $k T$ plasma temperature and $\gamma=m_{\mathrm{e}} / 2 k T$.

One obtains then

$$
\frac{1}{2} \sum_{i} b_{i i}=\frac{1}{2} \Gamma \sum_{i} \frac{\partial^{2} g}{\partial v_{i}^{2}}=\Gamma N_{\mathrm{e}}\left(\frac{\gamma}{\pi}\right)^{3 / 2} I
$$

where

$$
\boldsymbol{I}=\int \mathrm{d} \boldsymbol{v}^{\prime} e^{-\gamma v^{\prime}} \frac{1}{\left|\boldsymbol{v}-\boldsymbol{v}^{\prime}\right|}=\left(\frac{\pi}{\gamma}\right)^{3 / 2} \frac{1}{v} \operatorname{Erf}\left(\gamma^{1 / 2} v\right) .
$$

Furthermore,

$$
\begin{aligned}
\sum a_{i} v_{i} & =\Gamma \sum v_{i} \frac{\partial}{\partial v_{i}} h(\boldsymbol{v}) \\
& =\Gamma\left(1+\frac{m_{\mathrm{a}}}{m_{\mathrm{e}}}\right) N_{\mathrm{e}}\left(\frac{\gamma}{\tau}\right)^{3 / 2} v \frac{\partial I}{\partial v} .
\end{aligned}
$$

One thus obtains $\left(m_{\mathrm{a}} \gg m_{\mathrm{e}}\right)$

$$
\begin{aligned}
\frac{\mathrm{d} E}{\mathrm{~d} t}=\frac{4 \pi z^{2} e^{4}}{m_{\mathrm{e}}} \ln \Lambda & N_{\mathrm{e}}\left(\frac{2}{\sqrt{\pi}} \sqrt{\frac{m_{\mathrm{e}}}{2 k T}} \exp \left\{-\frac{m_{\mathrm{e}} v^{2}}{2 k T}\right\}\right. \\
& -\frac{1}{v} \operatorname{Erf}\left(\sqrt{\frac{m_{\mathrm{e}}}{2 k T}} v\right) .
\end{aligned}
$$

Now the range is defined as the distance travelled until averaged thermal energy $E_{\mathrm{th}}=\frac{3}{2} k T$ is reached. Thus if $E_{0}$ is the initial energy of the heavy particle the range $R$ is given by

$$
\begin{aligned}
R & =\int_{E_{\mathrm{a}}}^{E_{\mathrm{th}}} \frac{\mathrm{d} E}{-(\mathrm{d} E / \mathrm{d} x)}=\int_{E_{\mathrm{th}}}^{E_{0}} \frac{v \mathrm{~d} E}{\mathrm{~d} E / \mathrm{d} t} \\
& =\frac{m_{\mathrm{a}}}{m_{\mathrm{e}}}\left(\frac{k T}{e^{2}}\right)^{2} \frac{1}{N_{\mathrm{e}} \ln \Lambda \pi z^{2}} \int_{x_{1}}^{x_{2}} \frac{x^{3} \mathrm{~d} x}{f(x)}
\end{aligned}
$$

where

$$
\begin{aligned}
f(x) & =\operatorname{Erf}(x)-\frac{2}{\sqrt{\pi}} x e^{-x^{2}} \\
x_{1} & =\sqrt{\frac{3}{2} \frac{m_{\mathrm{e}}}{m_{\mathrm{a}}}}, \quad x_{2}=\sqrt{\frac{E_{0}}{k T} \frac{m_{\mathrm{e}}}{m_{\mathrm{a}}}} .
\end{aligned}
$$

As the temperature $k T$ increases we have $x_{2} \rightarrow x_{1}$. Thus the integral in (11) decreases to zero. However, the factor $(k T)^{2}$ increases much faster giving a range which will increase with $(k T)$.

\section{Quantum Electrodynamic Contribution}

As mentioned already there should be a theoretical correction to this formula since there is always some photon emission connected with scattering. The Feynman diagrams contributing to this are shown in Figure 1.
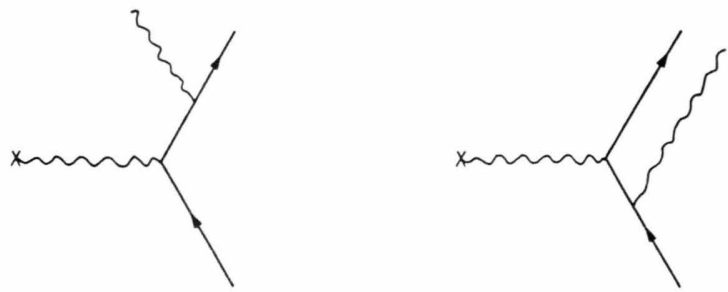

Fig. 1. Feynman Diagram showing electron scattering in external field together with photon emission.

Schwinger first calculated this contribution in a relativistic way. It has been also calculated with the help of Feynman rules by Bjorken and Drell ${ }^{11}$. In the non relativistic limit the corresponding crosssection is

$$
\left(\frac{\mathrm{d} \sigma}{\mathrm{d} \Omega}\right)_{i}=\left(\frac{\mathrm{d} \sigma}{\mathrm{d} \Omega}\right)_{\mathrm{c}} \frac{2 \alpha}{\pi} \frac{\mathrm{d} k}{k} \frac{4}{3} \beta^{2} \sin ^{2} \Theta / 2
$$

where $\alpha$ is the fine structure constant $(\alpha=1 / 137)$ and $\beta=u / c$ i. e. the relative velocity, $\theta$ the scattering angle, $(\mathrm{d} \sigma / \mathrm{d} \Omega)_{\mathrm{C}}$ is the Coulomb value. With respect to the photon frequency $\omega$ emitted: $\mathrm{d} k / k=\mathrm{d} \omega / \omega$. To find the contributing cross-section one has to integrate over all possible frequencies emitted say between $\omega_{\min }$ and $\omega_{\max }$

$$
\left(\frac{\mathrm{d} \sigma}{\mathrm{d} \Omega}\right)_{i}=\frac{2 \alpha}{3 \pi c^{2}} \frac{z^{2} e^{4}}{m_{\mathrm{e}}^{2}} \ln \left(\frac{\omega_{\max }}{\omega_{\min }}\right) \frac{1}{u^{2} \sin ^{2} \Theta / 2} .
$$

This expression is divergent for $\omega_{\min } \rightarrow 0$ which is the famous infrared divergence. We can use a cut. off for this and choose the "Bagge" frequency

$$
e^{2} / \lambda_{\mathrm{D}}=\hbar \omega_{\mathrm{B}}
$$

where $\lambda_{\mathrm{D}}$ is the Debye radias: $\lambda_{\mathrm{D}}=\sqrt{k T / 4 \pi N_{\mathrm{e}} e^{2}}$. Bagge ${ }^{4}$ first used this value for the binding energy correction to the Bethe-Bloch formula for the calculation of penetration depth of relativistic electrons in plasma. He obtained thereby ranges $R$ being of better consistency for explaining cosmic radiation and resulting in a remarkable agreement ${ }^{2}$ with the measured strong decrease of ranges in high density plasmas for relativistic electrons. For the maximum frequency emitted one can use the instantaneous energy of the alpha: $E=\hbar \omega_{\max }$. One thus obtains :

$$
\left(\frac{\mathrm{d} \sigma}{\mathrm{d} \Omega}\right)_{i}=\frac{2 \alpha}{3 \pi c^{2}} \frac{z^{2} e^{4}}{m_{\mathrm{e}}^{2}} \ln K \frac{1}{u^{2} \sin ^{2} \Theta / 2}
$$

where

$$
K=\frac{E}{e^{2}} \sqrt{\frac{k T}{4 \pi e^{2} N_{\mathrm{e}}}} .
$$


If one uses the sum of the differential cross-section, namely

$$
\frac{\mathrm{d} \sigma}{\mathrm{d} \Omega}=\left(\frac{\mathrm{d} \sigma}{\mathrm{d} \Omega}\right)_{\mathrm{c}}+\left(\frac{\mathrm{d} \sigma}{\mathrm{d} \Omega}\right)_{i}
$$

there will be additional terms for $a_{i}$ 's and $b_{i j}$ 's. We now work these out in detail: From the definitions

$$
\begin{aligned}
\boldsymbol{a}_{i} & =\int \mathrm{d} \boldsymbol{v}^{\prime} f_{\mathrm{e}}\left(\boldsymbol{v}^{\prime}\right)\{\Delta \boldsymbol{v}\}, \\
b_{i j} & =\int \mathrm{d} \boldsymbol{v}^{\prime} f_{\mathrm{e}}\left(\boldsymbol{v}^{\prime}\right)\left\{\Delta v_{i} \Delta v_{j}\right\} .
\end{aligned}
$$

Here $\{\Delta \boldsymbol{v}\}$ denotes the change per unit time in the velocity of the alpha by collision. Suppose $\boldsymbol{v}$ and $\boldsymbol{v}^{\prime}$ denote the velocity of the alpha and the electron before collision and $\boldsymbol{u}$ their relative velocity

$$
\begin{aligned}
& \boldsymbol{u}=\boldsymbol{v}-\boldsymbol{v}^{\prime}, \quad \boldsymbol{v}=\boldsymbol{V}+\frac{m_{\mathrm{e}}}{M} \boldsymbol{u}, \quad \boldsymbol{v}^{\prime}=\boldsymbol{V}-\frac{m_{\mathrm{a}}}{M} \boldsymbol{u}, \\
& M=m_{\mathrm{a}}+m_{\mathrm{e}}, \quad M \boldsymbol{V}=m_{\mathrm{a}} \boldsymbol{v}+m_{\mathrm{e}} \boldsymbol{v}^{\prime} .
\end{aligned}
$$

Now the change in $\boldsymbol{u}$ by collision, denoted $\Delta \boldsymbol{u}$, can be obtained considered Figure 2.

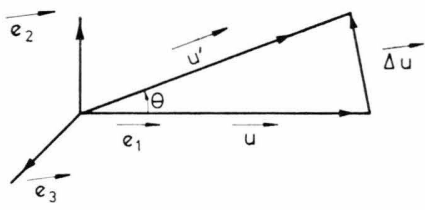

Fig. 2. Change in $\boldsymbol{u}$ by collision, $\Theta$ denoting scattering angle.

With notations as shown in the figure one has

$$
\begin{aligned}
|\Delta \boldsymbol{u}| & =2 u \sin \frac{\Theta}{2}, \\
\Delta \boldsymbol{u} & =|\Delta \boldsymbol{u}|\left[-\boldsymbol{e}_{1} \sin \frac{\Theta}{2}\right. \\
& \left.+\left(\boldsymbol{e}_{2} \cos \Phi+\boldsymbol{e}_{3} \sin \Phi\right) \cos \frac{\Theta}{2}\right] .
\end{aligned}
$$

Then

$$
\begin{aligned}
& \Delta \boldsymbol{v}=\frac{m_{\mathrm{e}}}{M} \Delta \boldsymbol{u} \cong \frac{m_{\mathrm{e}}}{m_{\mathrm{a}}} \Delta \boldsymbol{u}, \\
& \Delta v_{i} \Delta v_{j}=\left(\frac{m_{\mathrm{e}}}{m_{\mathrm{a}}}\right)^{2} \Delta u_{i} \Delta u_{j}, \\
& \{\Delta \boldsymbol{v}\}=\frac{m_{\mathrm{e}}}{m_{\mathrm{a}}} \int \mathrm{d} \Omega \frac{\mathrm{d} \sigma}{\mathrm{d} \Omega} u \Delta \boldsymbol{u}, \\
& (\mathrm{d} \Omega=\sin \Theta \mathrm{d} \Theta \mathrm{d} \Phi) \\
& \left\{\Delta v_{i} \Delta v_{j}\right\}=\left(\frac{m_{\mathrm{e}}}{m_{\mathrm{a}}}\right)^{2} \int \mathrm{d} \Omega \frac{\mathrm{d} \sigma}{\mathrm{d} \Omega} u \Delta u_{i} \Delta u_{j} .
\end{aligned}
$$

Substituting for the cross-section (13) one has

$$
\{\Delta \boldsymbol{v}\}=-\frac{m_{\mathrm{e}}}{m_{\mathrm{a}}} \frac{16 \alpha z^{2} e^{4}}{3 m_{\mathrm{e}}{ }^{2} c^{2}} \ln K \boldsymbol{e},
$$

$$
\begin{aligned}
\boldsymbol{a} & =\int \mathrm{d} \boldsymbol{v}^{\prime} f_{\mathrm{e}}\left(\boldsymbol{v}^{\prime}\right)\{\Delta \boldsymbol{v}\} \\
& =-\frac{m_{\mathrm{e}}}{m_{\mathrm{a}}} \frac{16 \alpha z^{2} e^{4}}{3 m_{\mathrm{e}}{ }^{2} c^{2}} \ln K \int \mathrm{d} \boldsymbol{v}^{\prime} f_{\mathrm{e}}\left(\boldsymbol{v}^{\prime}\right) \frac{\boldsymbol{v}-\boldsymbol{v}^{\prime}}{\left|\boldsymbol{v}-\boldsymbol{v}^{\prime}\right|} .
\end{aligned}
$$

Since

$$
\frac{\left|\boldsymbol{v}-\boldsymbol{v}^{\prime}\right|}{\boldsymbol{v}-\boldsymbol{v}^{\prime}}=\frac{\partial}{\partial \boldsymbol{v}}\left|\boldsymbol{v}-\boldsymbol{v}^{\prime}\right|
$$

one can write

$$
\boldsymbol{a}=-\frac{m_{\mathrm{e}}}{m_{\mathrm{a}}} \frac{16 \alpha z^{2} e^{4}}{3 m_{\mathrm{e}}{ }^{2} c^{2}} \ln K N_{\mathrm{e}}\left(\frac{\gamma}{\tau}\right)^{3 / 2} \frac{\partial I}{\partial \boldsymbol{v}}
$$

where

$$
\begin{gathered}
I=\int_{-\infty}^{+\infty} \mathrm{d} \boldsymbol{v}^{\prime} e^{-\gamma v^{\prime 2}}\left|v-v^{\prime}\right|=\frac{\pi}{\gamma^{3 / 2} v} \psi(v) \\
\psi(v)=\frac{v}{\sqrt{\gamma}} e^{-\gamma v^{2}}+\sqrt{\pi} \operatorname{Erf}(\sqrt{\gamma} v)\left(v^{2}+\frac{1}{2 \gamma}\right) .
\end{gathered}
$$

Thus

$\boldsymbol{a} \cdot \boldsymbol{v}=\frac{m_{\mathrm{e}}}{m_{\mathrm{a}}} \frac{16 \alpha z^{2} e^{4}}{3 m_{\mathrm{e}}^{2} c^{2}} \ln k\left(\frac{\gamma}{\tau}\right)^{\frac{3}{2}} N_{\mathrm{e}}\left[I-\frac{\pi}{\gamma^{3 / 2}} \Psi^{\prime}(v)\right]$.

Similarly

$$
\begin{aligned}
\frac{1}{2} \sum_{i} b_{i i} & =\frac{1}{2} \sum_{i} \int \mathrm{d} \boldsymbol{v}^{\prime} f_{\mathrm{e}}\left(\boldsymbol{v}^{\prime}\right)\left\{\Delta v_{i} \Delta v_{i}\right\} \\
& =\left(\frac{m_{\mathrm{e}}}{m_{\mathrm{a}}}\right)^{2} \frac{16 \alpha z^{2} e^{4}}{3 m_{\mathrm{e}}^{2} c^{2}} \ln K N_{\mathrm{e}}\left(\frac{\gamma}{\pi}\right)^{3 / 2} I
\end{aligned}
$$

giving $\sum_{i} \frac{1}{2} b_{i i} \ll \boldsymbol{a} \cdot \boldsymbol{v}$.

For the rate of energy less one therefore obtains

$$
\begin{aligned}
& \frac{\mathrm{d} E}{\mathrm{~d} t}=\frac{4 \pi z^{2} e^{4}}{m_{\mathrm{e}}} N_{\mathrm{e}} \ln A \frac{1}{v} \\
& \cdot\left[\frac{2}{\sqrt{\pi}} \sqrt{\frac{m_{\mathrm{e}}}{2 k T}} v \exp \left\{-\frac{m_{\mathrm{e}} v^{2}}{2 k T}\right\}-\operatorname{Erf}\left(\sqrt{\frac{m_{\mathrm{e}}}{2 k T}} v\right)\right] \\
& +\frac{16 \alpha z^{2} e^{4}}{3 \sqrt{\pi} m_{\mathrm{e}} c^{2}} \ln K N_{\mathrm{e}} \frac{1}{v} \frac{1}{\gamma}\left[\sqrt{\pi} \operatorname{Erf}\left(\sqrt{\frac{m_{\mathrm{e}}}{2 k T}} v\right)\right. \\
& \left.\cdot\left(-\frac{m_{\mathrm{e}}}{2 k T} v^{2}+\frac{1}{2}\right)-\sqrt{\frac{m_{\mathrm{e}}}{2 k T}} v \exp \left\{\frac{m_{\mathrm{e}} v^{2}}{2 k T}\right\}\right] .
\end{aligned}
$$

Using

$$
\frac{m_{\mathrm{e}} v^{2}}{2 k T}=\frac{m_{\mathrm{e}}}{m_{\mathrm{a}}} \frac{E}{k T}
$$

one obtains the following expression for the range:

$$
\begin{gathered}
R=\frac{m_{\mathrm{e}}}{m_{\mathrm{a}}} \frac{m_{\mathrm{e}} c^{2} k T}{N_{\mathrm{e}} z^{2} e^{4} \pi \ln \Lambda} \\
\int_{x_{1}}^{x_{2}} \frac{\mathrm{d} x}{\frac{2 m_{\mathrm{e}} c^{2}}{k T}\left[\frac{\operatorname{Erf}(\sqrt{x})}{x}-\frac{2 e^{-x}}{\sqrt{\pi x}}\right]+\frac{16 \alpha}{3} \frac{\ln K}{\ln \Lambda}} \\
\left\{\operatorname{Erf}(\sqrt{x})\left(-1+\frac{1}{2 x}\right)-\frac{e^{-x}}{\sqrt{\pi x}}\right\} \\
x_{1}=\frac{3}{2} \frac{m_{\mathrm{e}}}{m_{\mathrm{a}}}, \quad x_{2}=\frac{m_{\mathrm{e}}}{m_{\mathrm{a}}} \frac{E_{0}}{k T}
\end{gathered}
$$




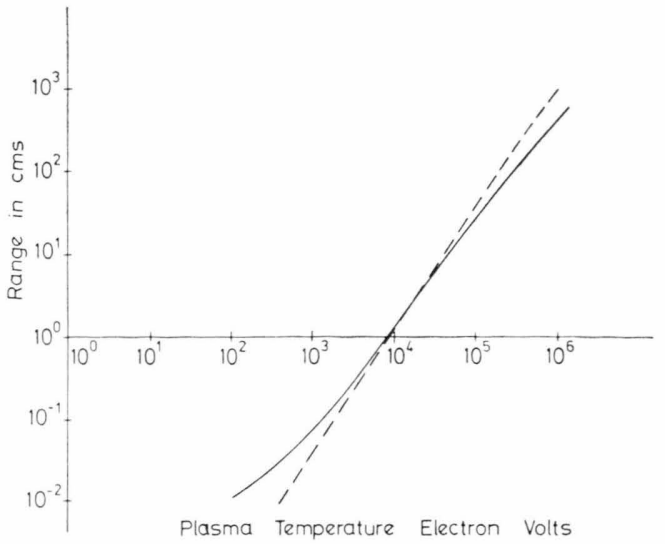

Fig. 3. Range of $3.6 \mathrm{MeV}$ alpha in DT plasma of solid state electron density. The dashed line shows values according to Winterberg formula.

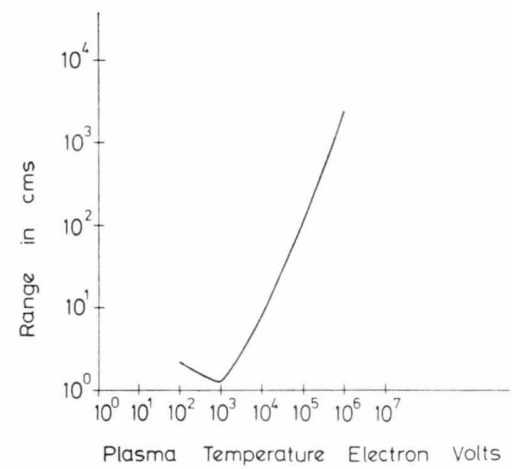

Fig. 4. Range of proton with initial energy $14.7 \mathrm{MeV}$ in DT plasma of solid state electron density.

if $k T$ is in electron volts one has for cgs units

$$
\begin{aligned}
R=\frac{m_{\mathrm{a}}}{m_{\mathrm{e}}} & \frac{10^{6} \times\left(6.9 \times 10^{6}\right)}{N_{\mathrm{e}} z^{2} 2 \pi \ln A}(k T) \\
& \cdot \int_{x_{1}}^{x_{2}} \frac{\mathrm{d} x}{\frac{10^{6}}{k T}\left[\frac{\operatorname{Erf}(\sqrt{x})}{x}-\frac{2 e^{-x}}{\sqrt{\pi x}}\right]} \\
& +\frac{16 \alpha}{3 \pi} \frac{\ln K}{\ln A}\left\{\operatorname{Erf}(\sqrt{x})\left(-1+\frac{1}{2 \alpha}\right)-\frac{e^{-x}}{\sqrt{\pi x}}\right\}
\end{aligned}
$$

where

$$
\ln k=\ln \left(\frac{m_{\mathrm{a}}}{m_{\mathrm{e}}} \frac{x}{\sqrt{4 \pi N_{\mathrm{e}}}}\left(\frac{k T}{e^{2}}\right)^{3 / 2}\right) .
$$

\section{Numerical Results}

In the adjoining figures we show the numerical range values as a function of plasma temperature for solid state electron density of alphas in hydro-

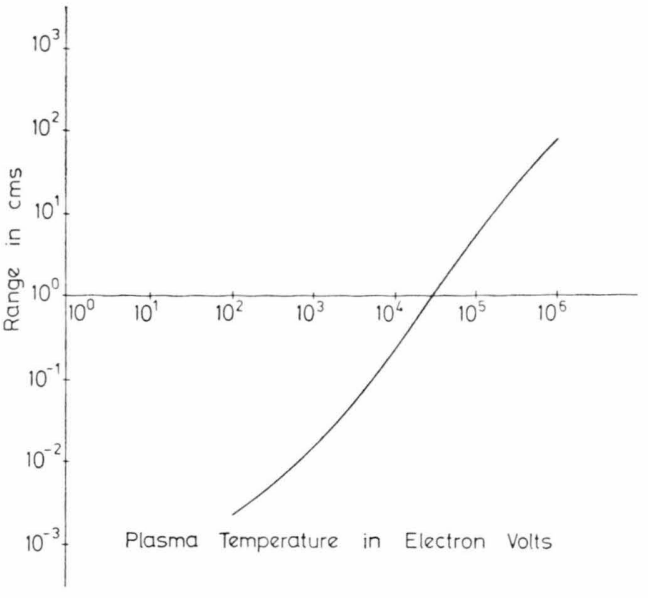

Fig. 5. Range of alpha with initial energy $2.894 \mathrm{MeV}$ in Hydrogen-Boron plasma of solid state electron density.

gen-boron and deuterium-tritium plasmas. We have also shown in Fig. 3 the deviation from our results of the Winterberg ${ }^{5}$ values usually used in literature. The above result differs from the Winterberg values at the lower and higher temperature ends.

One notes in Fig. 4 that for the case of $14.7 \mathrm{MeV}$ protons released by fusion in DT plasma the range first decreases with increasing temperature and subsequently increases. This behaviour is not shared by the alphas. The numerical value of the range is thus very susceptible to the ratio of the electron mass to the heavy particle mass as well as the initial heavy particle energy. Using the cut-off of the $\omega_{\max } /$ $\omega_{\min }$ as above, we can note that the numerical influence of the photon emissions during scattering is not substantial although this aspect has to be taken into account for a correct theory. This is mainly because of the enormous mass difference of the electrons and the heavy ions. However, for the case of external relativistic electron beam heating this could be of importance.

One should furthermore note that the larger the estimate for $\log \left(\omega_{\max } / \omega_{\min }\right)$ is chosen, the smaller the computed range would become. This is also feasible since emission of very soft photons during scattering should make this factor large. However, in the absence of experimental data for the range it is difficult to form any opinion on this aspect.

\section{Conclusion}

It is thus seen that usually accepted formula $R \sim T^{3 / 2}$ giving range as a function of plasma tem- 
perature for alphas in DT is not in general valid for other cases. The emission of photons during electromagnetic interaction of the heavy particle and the plasma electrons could have a substantial effect on

1 D. F. Düchs and D. Pfirsch, Proc. of Conf. on Plasma Physics and Controlled Nuclear Fusion, Vol. 1, Tokyo 1974, IAEA, p. 669.

2 J. R. Kerns et al., Bull. Amer. Phys. Soc. 17, 690 [1972].

3 E. Bagge, 13th Int. Cosmic Ray Conf. Denver, Aug 1973, The Origin of Cosmic Radiation (Thiemig, Munich) 1968.

4 E. Bagge and H. Hora, Atomkernenergie 24, 143 [1974].

5 F. Winterberg, Desert Research Institute Reprint Series, No. 64, March 1969.

- M. S. Chu, Ph.D. Thesis, Columbia University, 1971. the range if one chooses a large enough value for $\log \left(\omega_{\max } / \omega_{\min }\right)$ where $\omega_{\max }$ and $\omega_{\min }$ are the greatest and lowest photon frequencies emitted.

7 K. A. Brueckner and S. Jorna, Rev. Mod. Phys. 46, 325 [1974].

${ }^{8}$ K. A. Brueckner, H. Brysk, and R. S. Janda, J. Plasma Physics 11, 403 [1974].

9 R. P. Feynman, Quantum Electrodynamics, Benjamin, New York 1962, p. 150.

10 H. Tsuji et al, Nuclear Fusion 16, 287 [1976].

11 M. N. Rosenbluth et al., Phys. Rev. 107, 1 [1957].

12 J. Bjorken and S. Drell, Relativistic Quantum Mechanics, McGraw-Hill, New York 1964, p. 172. 